\title{
Recommendations for radioembolisation after liver surgery using yttrium-90 resin microspheres based on a survey of an international expert panel
}

\author{
Morsal Samim $^{1,2}$ • Linde M. van Veenendaal ${ }^{2}$ Manon N. G. J. A. Braat ${ }^{2}$. \\ Andor F. van den Hoven ${ }^{2} \cdot$ Richard Van Hillegersberg ${ }^{1} \cdot$ Bruno Sangro $^{3}$ • \\ Yung Hsiang $\mathrm{Kao}^{4}$ • Dave Liu ${ }^{5}$. John D. Louie ${ }^{6}$ - Daniel Y. Sze ${ }^{6}$. Steven C. Rose ${ }^{7}$. \\ Daniel B. Brown ${ }^{8} \cdot$ Hojjat Ahmadzadehfar ${ }^{9}$ Edward Kim ${ }^{10}$. \\ Maurice A. A. J. van den Bosch ${ }^{2}$ - Marnix G. E. H. Lam ${ }^{2}$
}

Received: 27 September 2016 / Revised: 15 March 2017 / Accepted: 11 May 2017 /Published online: 3 July 2017

(C) The Author(s) 2017. This article is an open access publication

\begin{abstract}
Introduction Guidelines on how to adjust activity in patients with a history of liver surgery who are undergoing yttrium-90 radioembolisation $\left({ }^{90} \mathrm{Y}-\mathrm{RE}\right)$ are lacking. The aim was to study the variability in activity prescription in these patients, between centres with extensive
\end{abstract}

Electronic supplementary material The online version of this article (doi:10.1007/s00330-017-4889-6) contains supplementary material, which is available to authorized users.

Morsal Samim

M.samim-3@umcutrecht.nl

1 Department of Surgery, University Medical Center Utrecht, Utrecht, The Netherlands

2 Department of Radiology and Nuclear Medicine, University Medical Center Utrecht, Utrecht, The Netherlands

3 Liver Unit, Clinica Universidad de Navarra-IDISNA and CIBEREHD, Pamplona, Spain

4 Department of Nuclear Medicine, Cabrini Hospital, Melbourne, Australia

5 Department of Radiology, Vancouver General Hospital. University of British Columbia, Vancouver, British Columbia, Canada

6 Division of Interventional Radiology, Stanford University Medical Center, Stanford, USA

7 Department of Radiology, University of California, San Diego, USA

8 Department of Radiology, Vanderbilt University, Medical Center North, Nashville, USA

9 Department of Nuclear Medicine, University Hospital Bonn, Bonn, Germany

10 Division of Vascular and Interventional Radiology, Icahn School of Medicine at Mount Sinai, New York, USA experience using resin microspheres ${ }^{90} \mathrm{Y}-\mathrm{RE}$, and to draw recommendations on activity prescription based on an expert consensus.

Methods The variability in activity prescription between centres was investigated by a survey of international experts in the field of ${ }^{90}$ Y-RE. Six representative postsurgical patients (i.e. comparable activity prescription, different outcome) were selected. Information on patients' disease characteristics and data needed for activity calculation was presented to the expert panel. Reported was the used method for activity prescription and whether, how and why activity reduction was found indicated.

Results Ten experts took part in the survey. Recommendations on activity reduction were highly variable between the expert panel. The median intra-patient range was $44 \mathrm{~Gy}$ (range 18-55 Gy). Reductions in prescribed activity were recommended in $68 \%$ of the cases. In consensus, a maximum $\mathrm{D}_{\text {Target }}$ of 50 Gy was recommended.

Conclusion With a current lack of guidelines, large variability in activity prescription in post-surgical patients undergoing ${ }^{90} \mathrm{Y}$-RE exists. In consensus, $\mathrm{D}_{\text {Target }} \leq 50 \mathrm{~Gy}$ is recommended.

Key points

- BSA method does not account for a decreased remnant liver volume after surgery.

- In post-surgical patients, a volume-based activity determination method is recommended.

- In post-surgical patients, a mean $D_{\text {Target }}$ of $\leq 50$ Gy should be aimed for.

Keywords Dosimetry $\cdot$ Radioembolisation $\cdot$ Liver . Guidelines $\cdot$ Yttrium 


\section{Introduction}

Liver surgery is often the preferred treatment option for patients with primary or secondary liver malignancies [1]. Unfortunately, primary curative liver resection is only feasible in a small minority of patients [1], and intrahepatic recurrence is commonly seen during follow-up [2]. When not amenable for repeated liver surgery, patients are often directed towards palliative therapies.

Hepatic ${ }^{90} \mathrm{Y}$ radioembolisation (RE) with resin microspheres (SIR-Spheres ${ }^{\circledR}$, Sirtex, Sydney, Australia) is an established treatment modality for both primary and secondary liver malignancies, and is considered to be safe and effective in patients with non-resectable hepatic malignancies [3].

Activity calculation [in Becquerel $(\mathrm{Bq})]$ for ${ }^{90} \mathrm{Y}-\mathrm{RE}$ is important for accurate treatment, in terms of an effective absorbed dose [in Gray (Gy)] to the tumour, while confirming a safe absorbed dose to the healthy liver tissue [3]. Ideally, activity calculation should be based on accurate and predictive pre-treatment dosimetry [4]. However, when using ${ }^{90} \mathrm{Y}$ resin microspheres, international guidelines have historically recommended the simple and semi-empirical body surface area (BSA) method for activity prescription [5-7].

Although the BSA method is popular due to its simplicity and is generally safe within the context of its original design [6], serious limitations for personalised dosimetry have come to light $[8,9]$. Studies have shown that BSA does not correlate well to the liver volume or tumour dosimetry, especially in situations of very high and very low tumour burden $[9,10]$. Logically, the lack of correlation between BSA and liver volume results in even higher ambiguity in patients with a history of liver surgery because of the reduced remnant liver volume (RLV) $[11,12]$. This scenario represents a significant number of patients currently receiving ${ }^{90} \mathrm{Y}-\mathrm{RE}$ [13-15], potentially leading to overdosing. In order to minimise the risk of clinically significant radioembolisation-induced liver disease (REILD), empirical dose reduction has been recommended for cases of small RLV [16]. Nevertheless, guidelines on how to adjust the prescribed activity in patients with a history of liver surgery are currently lacking.

The primary aim of this study was to study the variability in activity prescription in patients with a history of liver surgery between centres with extensive experience using resin microspheres ${ }^{90} \mathrm{Y}-\mathrm{RE}$, and to draw recommendations on activity prescription based on an expert panel survey.

\section{Materials and methods}

For this study, the patients' data was retrospectively collected. The study was reviewed by the institutional review board, and the requirement to obtain informed consent was waived. Records of patients undergoing ${ }^{90} \mathrm{Y}$-RE with resin microspheres between February 2009 and July 2015 were screened and patients with a history of liver surgery were selected.

\section{Expert panel}

For the survey on current clinical practice, a selection was made of post-surgical patients, based on their clinical history and post-treatment outcome. The selected patients presented a homogenous group in terms of primary malignancy and approximately comparable prescribed activity, but different surgical history and post-treatment outcome in terms of toxicity. The main goal of this selection was to reach sufficient patient variability in terms of remnant liver volumes and treatment outcome, in order to detect whether, how and why activity reduction was applied. All cases were presented to 10 international experts in the field of ${ }^{90} \mathrm{Y}$ RE, including 9 high-volume centres. The expert panel members were all internationally recognized experts on ${ }^{90} \mathrm{Y}$ RE. Included in the expert panel were six (interventional) radiologists, three nuclear medicine physicians (including the corresponding author) and one hepatologist. Patients' disease characteristics were summarised and representative imaging (CT and ${ }^{99 \mathrm{~m}} \mathrm{Tc}-\mathrm{MAA}$ ) were provided as well as all necessary data needed for activity calculation. The aim was to provide the expert panel members with all data needed for dosimetry calculation according to the current daily practice. They were blinded for treatment outcome.

\section{Endpoint}

The panel was primarily asked to prescribe activity based on expert opinion (along with an explanation of the rationale for any activity adjustment), and was blinded to the actual activity administration, and also to clinical outcome. The primary endpoint was the variability in prescribed activity for each case.

\section{Procedure}

All procedures were performed by experienced interventional radiologists (with at least 5 years of experience) according to standard recommendations [3]. The imaging workup prior to the ${ }^{90} \mathrm{Y}$-RE procedure required at least contrast-enhanced multiphase CT or MRI, with or without fluorine-18 $\left({ }^{18} \mathrm{~F}\right)$ fluorodeoxyglucose $\left({ }^{18} \mathrm{~F}-\mathrm{FDG}\right)$ positron emission tomography/ computed tomography (PET/CT). Technetium-99 m macroaggregated albumin $\left({ }^{99 \mathrm{~m}} \mathrm{Tc}-\mathrm{MAA}\right)$ was used for simulation imaging after coil embolisation of relevant hepatico-enteric vessels during preparatory angiography. Activity calculations were performed in compliance with international consensus guidelines 
and the package insert of resin microspheres $[3,7]$. The prescribed activity was calculated in GBq by the following equation $=$ BSA $\left(\mathrm{m}^{2}\right)-0.2+\%$ tumour involvement $/ 100$ [7].

In case of significant hepatopulmonary shunting ( $>30 \mathrm{~Gy}$ lung-absorbed dose), the prescribed activity was empirically reduced using standard reference tables provided by the manufacturer. The net administered activity was determined by correcting the prepared activity for residual activity in the $\mathrm{v}$ vial and tubing. Follow-up of patients consisted of evaluation of the treatment by means of telephone consultation at 2 weeks, clinical and laboratory examination at 4 weeks and imaging follow-up at 3 months. All adverse events were reported and toxicity was graded according to Common Terminology Criteria for Adverse Events (CTCAE) version 4.03 [17].

\section{Dosimetry}

We defined 'target-absorbed dose' $\left(\mathrm{D}_{\text {Target }}\right)$ as the mean absorbed dose (Gy) across the entire treated arterial territory, regardless of the natural microsphere heterogeneity between tumour and non-tumorous tissue. $\mathrm{D}_{\text {Target }}$ was retrospectively calculated using the medical internal radiation dose (MIRD) formula [18]. CT imaging was used to calculate the target volume, which was converted to mass ( $\mathrm{M}$ in $\mathrm{kg}$ ) using an assumed soft-tissue density of $1.06 \mathrm{~g} / \mathrm{ml}$. Homogeneous distribution of the microspheres in the target volume (including tumour and liver parenchyma) was assumed for calculating the $\mathrm{D}_{\text {Target }}$ utilising the following equation:

$D_{\text {Target }}(G y)=\left[A_{Y 90}(G B q) / M(k g)\right] * 50(J / G B q)$,

where $A_{Y 90}$ is the administered activity corrected for lung shunt.

\section{Statistical analysis}

Descriptive analyses were performed to summarize patient demographics and treatment characteristics. The variability in prescribed activity in each case was illustrated graphically. The variability was quantified by reporting the range of prescribed activity for each case and comparing it with the BSAbased prescribed activity. A commercial statistical software package (SPSS for Windows, version 19.0; SPSS Inc, Chicago, IL, USA) was used for data analysis.

\section{Results}

\section{Patient population}

Between February 2009 and July 2015, 187 consecutive patients with primary and secondary liver malignancies underwent ${ }^{90} \mathrm{Y}$-RE with resin microspheres in our clinic. Forty-three patients had a history of liver surgery varying from ablative therapy to an extended hemihepatectomy. Six patients were found eligible for the expert panel survey (Table 1). Five out of the six patients received ${ }^{90} \mathrm{Y}$-RE for colorectal liver metastases and one patient received treatment for cholangiocarcinoma. In all patients, the entire remnant liver was treated. The data used for activity calculation and presented to the expert panel is presented in Table 2.

For comparison between cases and experts, all prescribed activities were reported as target-absorbed dose or $\mathrm{D}_{\text {Target }}$, using prescribed activities and target volumes as described above. The actual prescribed activity ranged from 1.74 to $2.07 \mathrm{GBq}$ and resulted in a $\mathrm{D}_{\text {Target }}$ of 42-76 Gy. In three out of six patients (cases 3, 4 and 6), the delivered activity was reduced due to previous liver resection. The lowest net delivered $\mathrm{D}_{\text {Target }}$ was 40 Gy in case 2 with RLV of $2306 \mathrm{~mL}$, and the largest net delivered $\mathrm{D}_{\text {Target }}$ was 54 Gy in case 6 , with a RLV of $1091 \mathrm{~mL}$ (Table 2).

\section{Expert review and correlation}

The recommended activity adjustment for individual patients was highly variable and there was no consensus for any of the cases (Figure 1). The median intra-patient range (i.e. the highest minus the lowest recommended $\mathrm{D}_{\text {Target }}$ ) was 44 Gy (range 18-55 Gy). Reductions were reported 41 out of 60 times (68\%), no treatment at all in 2 out of 60 times $(3 \%)$, and there was one missing in the survey $(2 \%$; Table 3). Different methods were used to calculate the prescribed activity in cases of reduction. For most reductions $(17 / 41 ; 41 \%)$, the experts chose to empirically reduce the activity as a percentage of the prescribed activity based on the BSA method (median 20\%, range 10 $60 \%)$. In other cases $(15 / 41 ; 37 \%)$ a pre-set maximum safe $\mathrm{D}_{\text {Target }}$ was recommended (median 50 Gy, range 3555 Gy).The partition model was recommended 3 out of 41 times $(7 \%)$. In these cases, the recommended $\mathrm{D}_{\text {tumour }}$, $\mathrm{D}_{\text {Lung }}$ and maximum dose on healthy liver were given (median 40 Gy, range 40-54 Gy). Finally, in 6 out of 41 reductions $(15 \%)$, an empirically prescribed activity was recommended (median $46 \mathrm{~Gy}$, range 34-58 Gy; Table 3). Repeatedly, $>1$ reason for dose reduction was mentioned, resulting in a total of 65 reported reasons. Small RLV was the most frequent reason for activity reduction $(24 / 65 ; 37 \%)$. Other arguments for reduction were: previous systemic therapy $(16 / 65 ; 24 \%)$, low tumour burden $(10 / 65 ; 15 \%)$, findings of unfavourable microsphere biodistribution on ${ }^{99 \mathrm{~m}}$ Tc-MAA SPECT $(8 / 65 ; 12 \%)$, altered liver function $(5 / 65 ; 8 \%)$, previous radiotherapy $(1 / 65 ; 2 \%)$, and performance status $(1 / 65 ; 2 \%)$. 


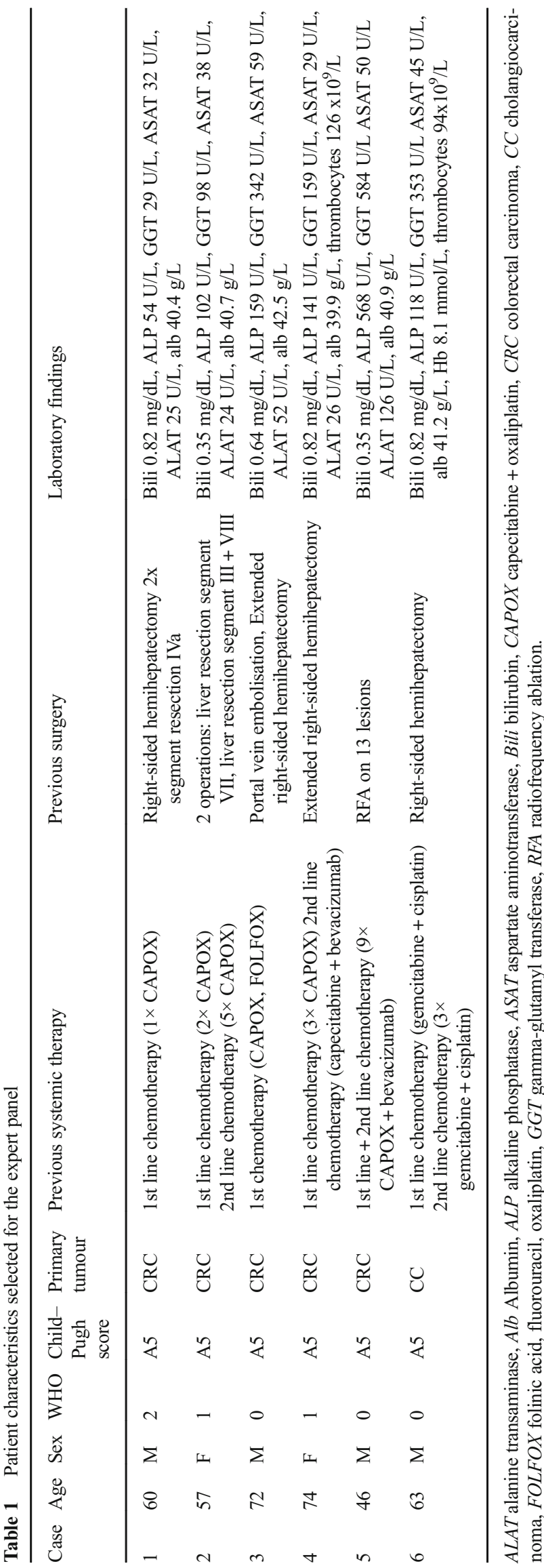

\section{Toxicity and outcome}

Post-treatment clinical symptoms were generally minor and included transient nausea, pain and fatigue related to the postembolisation syndrome (Table 4). REILD was seen in one patient (case 6) who developed grade 3 ascites with elevated liver biochemistry tests (with grade 1 hyperbilirubinemia at last measurement) and died 71 days after the treatment. The remaining cases developed only mild clinical and laboratory symptoms. With regards to disease outcome, case 2 was the first to develop progressive disease (within 3 months) and died 10 days after treatment due to progressive disease. Notably, the patient who developed REILD (case 6) had the smallest RLV and received the highest net $\mathrm{D}_{\text {Target }}$, while the latter patient (case 2) had the largest RLV and received the smallest $\mathrm{D}_{\text {Target }}$. The ${ }^{99 \mathrm{~m}}$ Tc-MAA SPECT images and the pre-procedural CT imaging of these two cases are shown in Figure 2.

\section{Discussion}

In general, ${ }^{90} \mathrm{Y}-\mathrm{RE}$ resin microspheres are safe for most patients with a history of liver surgery. However, the currently advocated BSA method for activity calculation does not incorporate the actual liver volume. In patients with extensive liver surgery, the remnant liver volume may have been significantly reduced, leading to potential overdosing in case this is not accounted for. Remarkably, there have been no publications on the effects of dosimetry on the outcome in terms of treatment response and toxicity in post-surgical patients. Since guidelines are lacking, the variability in used methods to reduce activity may be large. Consequently, administration of excessive activities to the normal liver parenchyma in postsurgical patients does occur, leading to fatal REILD in some cases. This study demonstrated that in current clinical practice, there is no consensus regarding the preferred method of adjustment of the prescribed activity in patients with a history of liver surgery. These findings raise some safety concerns for ${ }^{90}$ Y-RE after liver surgery.

Only a few studies have investigated the safety of ${ }^{90} \mathrm{Y}$-RE resin microspheres in patients with a history of liver surgery [13-15]. In all of these studies, activity calculation was based on the BSA method. It was, therefore, suggested that this method can safely be used in post-surgical patients. However, controversy remains, as it has been shown that the BSA model does not correlate well with liver volume, specifically in large and/or obese patients [8, 9]. Furthermore, patients that have undergone hepatic surgery pose an even greater challenge due to altered liver volume secondary to regeneration $[9,10]$, and due to a reduced liver functional reserve that may result in an increased risk of REILD. Significantly higher activity concentrations in the normal liver parenchyma can be measured in patients with pre-existent smaller liver 
Table 2 Data of patients selected for the expert panel that was used for activity calculation based on the BSA method

\begin{tabular}{|c|c|c|c|c|c|c|c|c|}
\hline Case & $\operatorname{BSA}\left(\mathrm{m}^{2}\right)$ & $\begin{array}{l}\text { Target } \\
\text { volume }(\mathrm{mL})\end{array}$ & $\begin{array}{l}\text { tumour } \\
\text { burden }(\%)\end{array}$ & $\begin{array}{l}\text { Lung } \\
\text { shunt }(\%)\end{array}$ & $\begin{array}{l}\text { Activity based on } \\
\text { BSA (GBq) }\end{array}$ & $\begin{array}{l}\text { Target dose based } \\
\text { on BSA (Gy) }\end{array}$ & $\begin{array}{l}\text { Target dose in case } \\
\text { of reduction }(\mathrm{Gy})^{\#}\end{array}$ & $\begin{array}{l}\text { Net delivered } \\
\text { dose }(\mathrm{Gy})^{*}\end{array}$ \\
\hline 1 & 2.06 & 1654 & 3 & 8 & 1.89 & 54 & - & 47 \\
\hline 2 & 2.17 & 2306 & 10 & 1 & 2.07 & 42 & - & 40 \\
\hline 3 & 1.91 & 1091 & 5 & 3 & 1.76 & 76 & 50 & 44 \\
\hline 4 & 1.79 & 1218 & 15 & 5 & 1.74 & 70 & 50 & 43 \\
\hline 5 & 2.05 & 1894 & 6 & 4 & 1.91 & 48 & - & 45 \\
\hline 6 & 1.90 & 1091 & 6 & 4 & 1.76 & 76 & 57 & 54 \\
\hline
\end{tabular}

\# The net target absorbed dose that was delivered after reduction: 50 Gy target absorbed dose in case 3 and 4, 25\% reduction in case 6 .

* The net delivered target absorbed dose is the mean absorbed dose (Gy) across the entire treated arterial territory calculated by the net administered activity.

Fig. 1 The target absorbed dose (Gy) as recommended by the expert panel for each case $(\mathrm{A}-\mathrm{F})$. The median (and range) recommended absorbed dose for each case was $49 \mathrm{~Gy}$, range $32-54$ (A), 42 Gy, range 0-55 Gy (B), $55 \mathrm{~Gy}$, range $30-76$ Gy $(\mathrm{C})$, $53 \mathrm{~Gy}$, range 50-68 Gy (D), $46 \mathrm{~Gy}$, range $0-50 \mathrm{~Gy}(\mathrm{E})$ and 59 Gy, range 35-76 Gy (F). Note: 0 Gy means that the expert advised not to treat that specific patient at all.
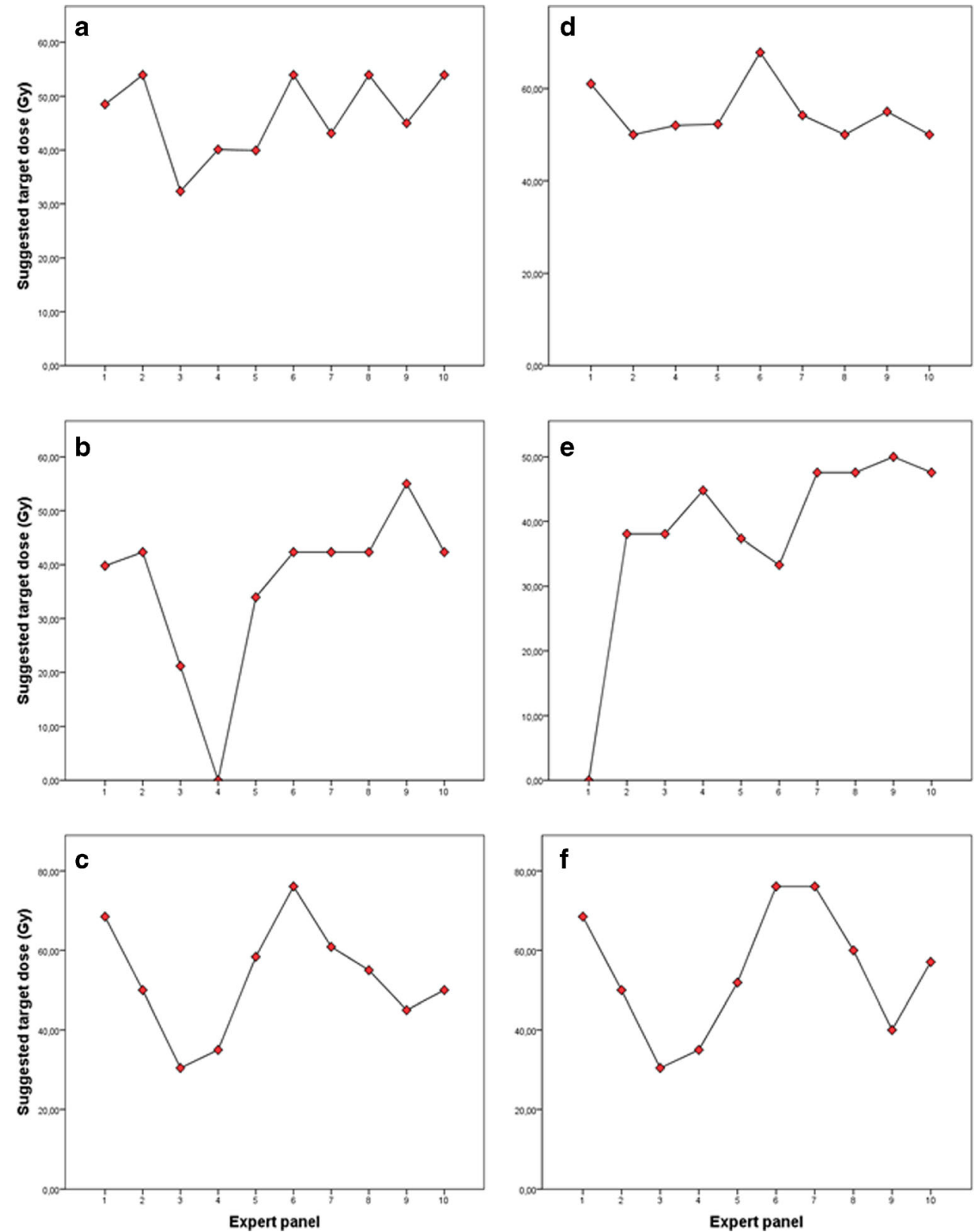
Table 3 The prescribed $\mathrm{D}_{\text {Target }}$ (Gy) for each case as advised by the expert panel

\begin{tabular}{llllllll}
\hline & & Case 1 & Case 2 & Case 3 & Case 4 & Case 5 & Case 6 \\
\hline Expert 1 & Type & PC & PC & PC & PC & No treatment & PC \\
& $\mathrm{D}_{\text {Target }}(\mathrm{Gy})$ & 49 & 39 & 68 & 63 & & 68 \\
Expert 2 & Type & NR & NR & MIRD & MIRD & PC & MIRD \\
& $\mathrm{D}_{\text {Target }}(\mathrm{Gy})$ & 54 & 42 & 50 & 50 & 38 & 50 \\
Expert 3 & Type & PC & PC & PC & Missing & PC & PC \\
& $\mathrm{D}_{\text {Target }}(\mathrm{Gy})$ & 32 & 21 & 30 & & 38 & 30 \\
Expert 4 & Type & PM & No & MIRD & PM & PM & MIRD \\
& $\mathrm{D}_{\text {Target }}(\mathrm{Gy})$ & 40 & Treatment & 35 & 54 & 40 & 35 \\
Expert 5 & Type & EPA & EPA & EPA & EPA & EPA & EPA \\
& $\mathrm{D}_{\text {Target }}(\mathrm{Gy})$ & 40 & 34 & 58 & 54 & 37 & 52 \\
Expert 6 & Type & NR & NR & NR & NR & PC & NR \\
& $\mathrm{D}_{\text {Target }}(\mathrm{Gy})$ & 54 & 42 & 76 & 70 & 34 & 76 \\
Expert 7 & Type & PC & NR & PC & PC & NR & NR \\
& $\mathrm{D}_{\text {Target }}(\mathrm{Gy})$ & 43 & 42 & 61 & 56 & 48 & 76 \\
Expert 8 & Type & NR & NR & MIRD & MIRD & NR & PC \\
& $\mathrm{D}_{\text {Target }}(\mathrm{Gy})$ & 54 & 42 & $50-60$ & $50-60$ & 48 & 60 \\
Expert 9 & Type & MIRD & MIRD & MIRD & MIRD & MIRD & MIRD \\
& $\mathrm{D}_{\text {Target }}(\mathrm{Gy})$ & 45 & 55 & 45 & 55 & 50 & 40 \\
Expert 10 & Type & NR & NR & MIRD & MIRD & NR & PC \\
& $\mathrm{D}_{\text {Target }}(\mathrm{Gy})$ & 54 & 42 & 50 & 50 & 48 & 57 \\
\hline
\end{tabular}

EPA Empirically prescribed activity, MIRD Maximum safe whole liver absorbed dose $\left(\mathrm{D}_{\text {Target }}\right)$ based on Medical Internal Radiation Dose (MIRD) dosimetry, $N R$ no reduction was recommended, $P C$ Empirical reduction of the activity as a percentage of the prescribed activity based on the BSA method, $P M$ Partition model with prescribed $\mathrm{D}_{\text {Tumour }}, \mathrm{D}_{\mathrm{HL}}$ and $\mathrm{D}_{\text {Lung }}$ (the tumour to non-tumour ratio was assumed). volumes, or reduced liver volumes [10]. The cases of the expert panel survey showed significant toxicity in case of a high $\mathrm{D}_{\text {Target }}(\mathrm{Gy})$ in a patient with a relatively small RLV (i.e. overdosing), and suggested underdosing in case of a small $\mathrm{D}_{\text {Target }}(\mathrm{Gy})$ in patients with a relatively large RLV (and comparable BSA). This finding again illustrates the lack of correlation between BSA and the (remnant) liver volume.
The results of the survey illustrated the lack of consensus regarding the preferred method of activity reduction in any of the cases. Moreover, different methods were used for activity adjustment. In a high percentage of cases, the expert panel agreed on the fact that a low RLV is an argument for careful use of the BSA method, due to the high risk of an overdose. Preferably, a specific $\mathrm{D}_{\text {Target }}$ was prescribed (frequently $\pm 50 \mathrm{~Gy}$ ), and in some
Table 4 Reported adverse events within 3 months following the ${ }^{90} \mathrm{Y}$ RE procedure according to the CTCAE version 4.03 for the six cases presented to the expert panel

\begin{tabular}{lll}
\hline $\begin{array}{l}\text { Adverse } \\
\text { events }\end{array}$ & Laboratory toxicity* & Clinical toxicity \\
\hline Case 1 & $\begin{array}{c}\text { ALP (+1 CTC) GGT (+3 CTC) ASAT (+1 CTC) } \\
\text { ALAT (+1 CTC) }\end{array}$ & Fatigue (CTC 1) Nausea (CTC 1) \\
Case 2 & ALP (+1 CTC) GGT (+1 CTC) & $\begin{array}{c}\text { Fatigue (CTC 2) Nausea (CTC 1) Pain } \\
\text { (CTC 2) }\end{array}$ \\
Case 3 & Bili (+2 CTC) ALP (+1 CTC) ASAT (+1 CTC) & $\begin{array}{c}\text { Fatigue (CTC 1) Nausea (CTC 1) Pain } \\
\text { (CTC 1) }\end{array}$ \\
Case 4 & GGT (+1 CTC) ASAT (+1 CTC) ALAT (+1 CTC) & $\begin{array}{c}\text { None } \\
\text { Case } 5\end{array}$ \\
Bili (+1 CTC) & $\begin{array}{c}\text { Fatigue (CTC 1) Pain (CTC 1) } \\
\text { Jaundice }\end{array}$ \\
Case 6 & Bili (+1 CTC) ALP (+1 CTC) ALAT (+1 CTC) & Pain (CTC 2) Ascites (CTC 2) \\
& Albumin (+2 CTC) & \\
\hline
\end{tabular}

ALAT alanine transaminase, ALP alkaline phosphatase, ASAT aspartate aminotransferase; Bili: bilirubin, CTC common toxicity criteria, GGT gamma-glutamyl transferase.

*Laboratory toxicity is reported as highest increase in CTC toxicity grade compared to baseline CTC grade (+1 etc.) 
Fig. 2 The ${ }^{99 \mathrm{~m}}$ Tc-MAA SPECT (A and $\mathrm{C}$ ) and the pre-procedural $\mathrm{CT}$ imaging (B and $\mathrm{D})$ of, respectively, cases 2 and 6 as presented to the expert panel along with following information: the ${ }^{99 \mathrm{~m}}$ Tc-MAA SPECT of case 2 shows low activity around the largest lesion in segment VIII and relatively high uptake in the healthy liver parenchyma after injection of MAA in the left hepatic artery (A and B). In case 6 , the ${ }^{99 \mathrm{~m}}$ Tc-MAA SPECT and the pre-procedural $\mathrm{CT}$ imaging show distribution of the activity throughout the remnant liver, although with lack of activity uptake around the largest lesion as depicted by the red circle $(\mathrm{C}$ and $\mathrm{D})$.
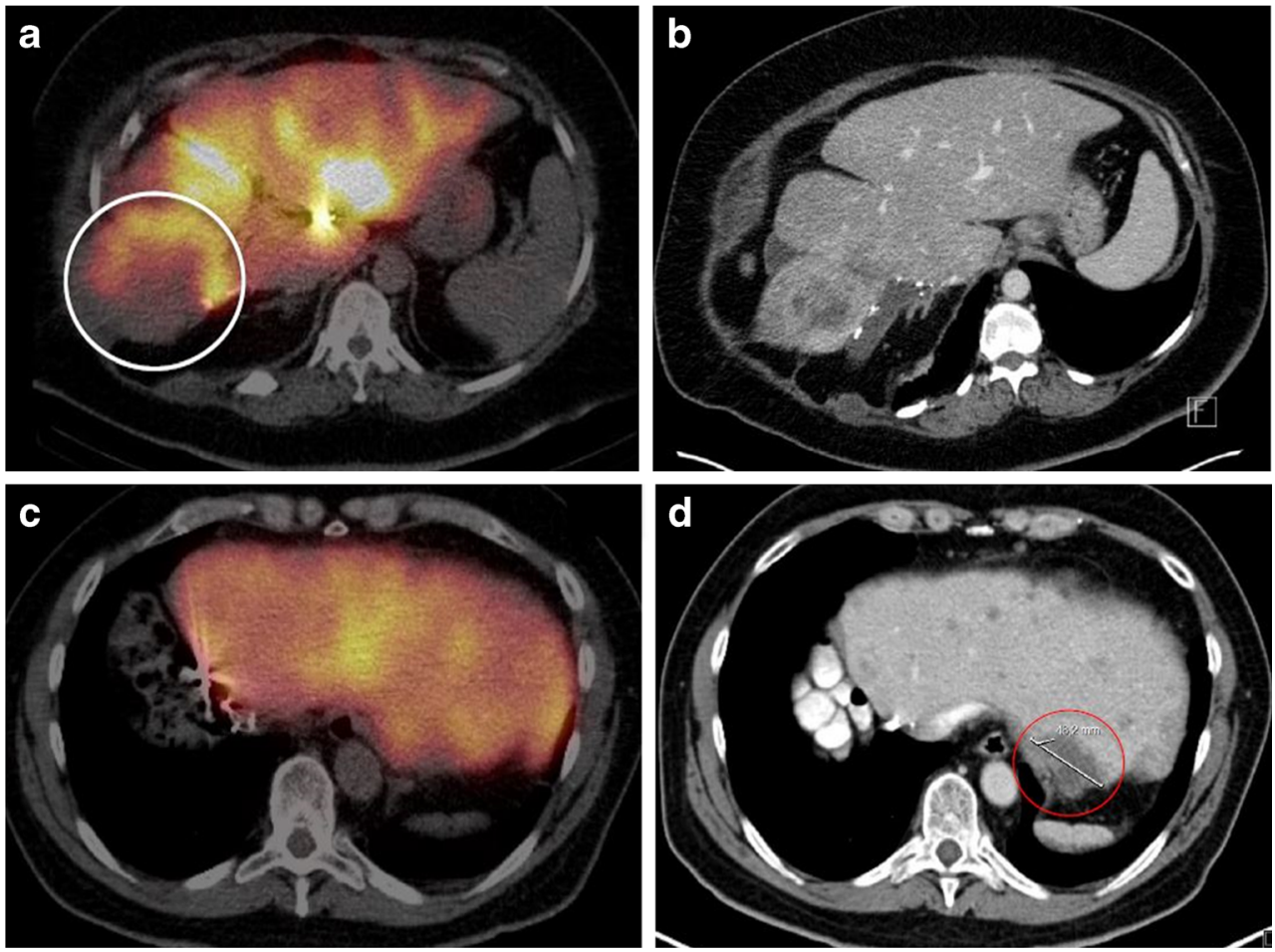

cases, the $\mathrm{D}_{\text {Target }}$ was further specified in terms of absorbed dose to the tumour tissue, non-tumour tissue and the lungs, according to the 'partition model' which is based on MIRD macrodosimetry $[6,18,19]$. Clinical explanations may be provided for the differences in activity prescription provided by the expert panel. The patients who are eligible for ${ }^{90} \mathrm{Y}-\mathrm{RE}$ treatment represent a heterogeneous group with distinct disease characteristics. There is significant diversity in the patients' medical histories, such as prior chemotherapy, which is known to increase the risk of REILD [20]. Also, tumour vascularity may differ, which influences the microsphere biodistribution of the delivered activity to tumour and non-tumour tissue [8]. It has been mentioned previously that the BSA method neglects the inter-patient variability in tumour-to-normal liver tissue microsphere biodistribution [6, 19], a possible argument for activity adjustment. Lastly, the extent of liver surgery differs highly, resulting in large differences between remnant liver volumes.

A limitation of this study is the retrospective selection of patients in the survey. However, the selected patients were representative for daily practice, with risk of under- and overdosing in patients with similar prescribed activity (based on the BSA method) but distinct RLV and DTarget. Hence, the lack of guidelines for dosimetry, especially in this patient group, directly affects clinical outcome and should be investigated more thoroughly.

Based on these results, the blind application of the BSA method for activity prescription in patients with previous liver surgery is discouraged. Based on the reports of the expert panel, the following consensus recommendation can be made; for activity calculation of ${ }^{90} \mathrm{Y}-\mathrm{RE}$ using resin microspheres, a volume-based activity calculation algorithm is recommended, such as the MIRD method that takes RLV into account. A maximum $\mathrm{D}_{\text {Target }}$ of 50 Gy seems a safe interim recommendation, with further reduction in the case of relatively hypovascular tumours, dismal baseline liver function, or a heavily pre-treated patient. The partition model may also warrant further consideration and research as it theoretically takes into account the differential activity biodistribution to both the tumour and the normal liver tissue [19, 21]. As regards to a recommended safe cut-off value for the dose on healthy liver tissue, different possible safe cut-off points have been reported $[6,22]$. However, in view of the applicability of the partition model for individualised dosimetry, more research on this topic is essential. Finally, in order to facilitate patient-specific dosimetry of ${ }^{90} \mathrm{Y}-\mathrm{RE}$ treatment and future research on this topic, accessible tools have been developed [23].

\section{Conclusion}

Caution should be taken when using the BSA method for activity prescription in patients with previous liver resection. Based on the survey of the expert panel, an interim recommendation is to use a volume-based activity determination method such as the MIRD-based model instead, aiming for a mean absorbed dose across the target arterial territory $\left(\mathrm{D}_{\text {Target }}\right)$ 
of $\leq 50 \mathrm{~Gy}$, until the emergence of more robust dosimetric data. Partition modelling should lead to further refinement in personalised activity calculation based on healthy liver and lungabsorbed dose thresholds.

Acknowledgements MS has received a personal grant-Alexander Suerman Stipendium — for her MD-PhD.

\section{Compliance with ethical standards}

Guarantor The scientific guarantor of this publication is M. Lam.

Conflict of interest The authors of this manuscript declare relationships with the following companies:

MS is a speaker for Sirtex. BS, DL and MGL are consultants for Sirtex. BS, DYS and MGL are consultants for BTG. DL and EK are on the advisory board for BTG. JDL is consultant for Biocompatibles, Cardia Health and Medtronic. DBB is consultant for Cook Medical. EK is on the advisory board for Boston Scientific. SCR is on the scientific advisory board for Surefire Medical and consultant for Embolx, Guerbet and XL SciTech. YHK has received a research funding support from Sirtex.

Funding The authors state that this work has not received any funding.

Statistics and biometry One of the authors has significant statistical expertise (M. Samim).

Informed consent Written informed consent was waived by the Institutional Review Board.

Ethical approval Institutional Review Board approval was not applicable for this study. The ethical committee reviewed the study protocol and confirmed that the Medical Research Involving Human Subjects Act (WMO) does not apply to our study and that, therefore, an official approval of this study by the MREC UMC Utrecht was not required under the WMO.

\section{Methodology \\ - retrospective \\ - observational \\ - performed at one institution}

Open Access This article is distributed under the terms of the Creative Commons Attribution 4.0 International License (http:// creativecommons.org/licenses/by/4.0/), which permits unrestricted use, distribution, and reproduction in any medium, provided you give appropriate credit to the original author(s) and the source, provide a link to the Creative Commons license, and indicate if changes were made.

\section{References}

1. Khatri VP, Petrelli NJ, Belghiti J (2005) Extending the frontiers of surgical therapy for hepatic colorectal metastases: Is there a limit? J Clin Oncol 23:8490-8499

2. de Jong M, Pulitano C, Ribero D et al (2009) Rates and patterns of recurrence following curative intent surgery for colorectal liver metastasis: An international multi-institutional analysis of 1669 patients. Ann Surg 250:440-8

3. Kennedy A (2014) Radioembolisation of hepatic tumors. J Gastrointest Oncol 5:178-189
4. Cremonesi M, Chiesa C, Strigari L et al (2014) Radioembolisation of hepatic lesions from a radiobiology and dosimetric perspective. Front Oncol 4:210-210

5. Giammarile F, Bodei L, Chiesa C et al (2011) EANM procedure guideline for the treatment of liver cancer and liver metastases with intra-arterial radioactive compounds. Eur J Nucl Med Mol Imaging 38:1393-1406

6. Kao YH, Lichtenstein M (2016) Origin, dosimetric effect and clinical limitations of the semi-empirical body surface area method for radioembolisation using yttrium-90 resin microspheres. J Med Imaging Radiat Oncol. doi:10.1111/1754-9485.12449

7. (2016) Package Insert for SIR-SpheresÂß Y-90 resin microspheres (2014)

8. Kao YH, Tan EH, Ng CE, Goh SW (2011) Clinical implications of the body surface area method versus partition model dosimetry for yttrium- 90 radioembolisation using resin microspheres: A technical review. Ann Nucl Med 25:455-61

9. Lam MGEH, Louie JD, Abdelmaksoud MHK, Ga F, Cho-Phan C, Sze DY (2014) Limitations of body surface area-based activity calculation for radioembolisation of hepatic metastases in colorectal cancer. J Vasc Interv Radiol 25:1085-1093

10. Grosser OS, Gerhard U, Furth C et al (2012) Intrahepatic activity distribution in radioembolisation with yttrium-90-labeled resin microspheres using the body surface area method-a less than perfect model. J Vasc Interv Radiol 26:1615-1621

11. Kokudo N, Tada K, Seki M et al (2001) Anatomical major resection versus nonanatomical limited resection for liver metastases from colorectal carcinoma. Am J Surg 181:153-9

12. Garden OJ, Rees M, Poston GJ et al (2006) Guidelines for resection of colorectal cancer liver metastases. Gut 55:iii1-iii8

13. Bester L, Feitelson S, Milner B, Chua TC, Morris DL (2013) Impact of prior hepatectomy on the safety and efficacy of radioembolisation with yttrium-90 microspheres for patients with unresectable liver tumors. Am J Clin Oncol 00:1-7

14. Geisel D, Powerski MJ, Schnapauff D et al (2014) No infectious hepatic complications following radioembolisation with $90 \mathrm{Y} \mathrm{mi-}$ crospheres in patients with biliodigestive anastomosis. Anticancer Res 34:4315-4321

15. Kessler J, Lewis A, Gagandeep S, Ituarte PHG, Park JJ (2015) Radioembolisation following liver resection: Safety and dosing considerations. J Vasc Interv Radiol:1-6. Doi:10.1016/j.jvir.2015.09.017

16. Kennedy A, Nag S, Salem R et al (2007) Recommendations for radioembolisation of hepatic malignancies using yttrium-90 microsphere brachytherapy: A consensus panel report from the radioembolisation brachytherapy oncology consortium. Int $J$ Radiat Oncol Biol Phys 68:13-23

17. Anonymous (2010) Common terminology criteria for adverse events (CTCAE). 2017

18. Gulec SA, Mesoloras G, Stabin M (2006) Dosimetric techniques in 90Y-microsphere therapy of liver cancer: The MIRD equations for dose calculations. J Nucl Med 47:1209-1211

19. Lau WY, Kennedy AS, Kim YH et al (2012) Patient selection and activity planning guide for selective internal radiotherapy with yttrium-90 resin microspheres. Int J Radiat Oncol Biol Phys 82:401-407

20. Gil-Alzugaray B, Chopitea A, Inarrairaegui M et al (2013) Prognostic factors and prevention of radioembolisation-induced liver disease. Hepatology 57:1078-1087

21. Ho S, Lau WY, Leung TW et al (1996) Partition model for estimating radiation doses from yttrium- 90 microspheres in treating hepatic tumours. Eur J Nucl Med 23:947-952

22. Lam MG, Goris ML, Iagaru AH, Mittra ES, Louie JD, Sze DY (2013) Prognostic utility of 90 Y radioembolisation dosimetry based on fusion $99 \mathrm{mTc}$-macroaggregated albumin-99mTc-sulfur colloid SPECT. J Nucl Med 54:2055-2061

23. (2016) University of British Columbia Â@ 2015-2016 Dave Liu (2016) DAVYR Y90 radioembolisation dosimetry calculator 